\title{
Elastic scattering of surface plasmon polaritons: Modeling and experiment
}

\section{Bozhevolnyi, Sergey I.; Coello, V.}

\section{Published in:}

Physical Review B Condensed Matter

Link to article, DOI:

10.1103/PhysRevB.58.10899

Publication date:

1998

Document Version

Publisher's PDF, also known as Version of record

Link back to DTU Orbit

\section{Citation (APA):}

Bozhevolnyi, S. I., \& Coello, V. (1998). Elastic scattering of surface plasmon polaritons: Modeling and experiment. Physical Review B Condensed Matter, 58(16), 10899-10910.

https://doi.org/10.1103/PhysRevB.58.10899

\section{General rights}

Copyright and moral rights for the publications made accessible in the public portal are retained by the authors and/or other copyright owners and it is a condition of accessing publications that users recognise and abide by the legal requirements associated with these rights.

- Users may download and print one copy of any publication from the public portal for the purpose of private study or research.

- You may not further distribute the material or use it for any profit-making activity or commercial gain

- You may freely distribute the URL identifying the publication in the public portal 


\title{
Elastic scattering of surface plasmon polaritons: Modeling and experiment
}

\author{
Sergey I. Bozhevolnyi \\ Mikroelektronik Centret, Technical University of Denmark, Building 345 east, DK-2800 Lyngby, Denmark \\ Victor Coello \\ Institute of Physics, Aalborg University, Pontoppidanstraede 103, DK-9220 Aalborg, Denmark
}

(Received 9 March 1998)

\begin{abstract}
Elastic (in-plane) scattering of surface plasmon polaritons (SPP's) is modeled by considering isotropic pointlike scatterers whose responses to the incident SPP field are phenomenologically related to their effective polarizabilities. Numerical simulations of single, double, and multiple scattering are presented for randomly situated scatterers showing the interplay between different orders of scattering and localization phenomena. Correlation between the scattering regimes and spatial Fourier spectra of the corresponding SPP intensity distributions is considered. Various optical microcomponents (e.g., straight and curved micromirrors) formed by sets of point scatterers are also simulated, and the stability and dispersion of their characteristics are investigated. The appropriate experimental results for SPP scattering by both random and specially configured sets of microscatterers are reported for two excitation wavelengths $(594$ and $633 \mathrm{~nm}$ ) and different metal (silver and gold) films. The near-field optical images obtained are related to the calculated SPP intensity distributions demonstrating that the model developed can be successfully used in studies of SPP elastic scattering, e.g., to design the microcomponents for SPP's. [S0163-1829(98)07039-8]
\end{abstract}

\section{INTRODUCTION}

Surface plasmon polaritons (SPP's), i.e., collective oscillations of the surface electron density, can be excited along an interface between dielectric and metal, and represent quasi-two-dimensional waves with amplitudes decaying exponentially into either of the neighbor media. ${ }^{1}$ Having the field maximum at the interface, SPP's are extremely sensitive to surface properties, a circumstance that attracted a great deal of attention to SPP's from researchers in both basic and applied areas of physics. Many fundamental properties of SPP's have been studied theoretically and experimentally, and various applications (mostly for surface sensing) have been suggested during the first two decades of extensive investigations. ${ }^{1}$ The development of near-field optical microscopy ${ }^{2}$ has led to the significant increase in the interest to the research in this area, because near-field techniques open up an exciting possibility to directly observe various SPP properties (from resonance field enhancement to propagation and scattering) and to investigate these properties with the spatial resolution on a nanometer scale. ${ }^{3}$

Different near-field techniques have been employed in the aforementioned studies of SPP's (overviews can be found in recent papers by Bozhevolnyi, Smolyaninov, and Zayats ${ }^{4}$ and Smolyaninov et al. ${ }^{5}$ ). However, a photon scanning tunneling microscope (PSTM), ${ }^{6}$ in which an uncoated fiber tip is used to detect an evanescent field of the light being totally internally reflected at the sample surface, seems to be the most suitable technique for local and unobtrusive probing of the SPP field. It has been theoretically argued that, due to the relatively low refractive index of optical fiber, such a tip can be within certain approximations considered as a nonperturbative probe of the electric-field intensity. ${ }^{7}$ Lately our detailed experimental investigations demonstrated convincingly that, for the SPP being resonantly excited at a relatively smooth surface, near-field optical images (obtained with an uncoated fiber tip) can be indeed directly related to the intensity distributions of the total SPP field, i.e., field of the excited and (in-plane) scattered SPP's. ${ }^{8}$ Hereafter, SPP scattering in the surface plane is denoted as elastic SPP scattering, since SPP scattering into a free space is an unwanted process leading to the additional (radiative) losses experienced by the SPP.

The progress in both understanding of local SPP properties and techniques available for their studies stimulated research in various directions, one of them being investigations of possibility for local controlling and directing SPP's, especially in the surface plane. Thus, local SPP excitation has been demonstrated by using a metal coated tapered fiber probe as a radiation source ${ }^{9}$ and by changing coupling conditions (in the Kretschmann configuration) with individual surface defects created by a direct-write PSTM-based technique. ${ }^{5,10}$ In the latter work, elastic SPP scattering (in the surface plane) by an artificial surface defect has also been attempted but with poor results. More pronounced elastic scattering of SPP's has been observed with artificial surface structures produced by means of electron-beam lithography. ${ }^{11}$ It is clear that an elastic microscatterer should be relatively large and with smooth boundaries in order to maximize the scatterer's strength while preserving an adiabatic perturbation. We believe that film material modification ${ }^{5,10}$ can hardly be used for fabricating such a scatterer as the influence of material properties on the SPP characteristics is known to be very strong. ${ }^{1}$ Quite recently, we have suggested a technique that relies on the local deformation of a metal film surface with an uncoated fiber tip, and demonstrated its suitability for fabricating various sets of elastic microscatterers that act as micro-optical components (e.g., micromirrors and microcavities) for SPP's. ${ }^{12}$ Even though the main principle of our technique is simple, a 
proper modeling is desirable to develop a better understanding of operation of this kind of microcomponents. Dispersion of microcomponents and the stability of their characteristics are among the issues to be clarified (preferably both experimentally and theoretically).

Another research direction is concerned with elastic SPP scattering by randomly situated scatterers and the resulting localization phenomena, viz., weak and strong (Anderson) localization of SPP's. ${ }^{13}$ Localization phenomena are caused by interference in multiple elastic scattering that results in the reduction of the mean free path. ${ }^{14}$ Actually, strong localization implies that the mean free path vanishes and propagation no longer exists, and this requires rather efficient multiple scattering. Weak localization (or enhanced backscattering) arises from a constructive interference (in the backscattering direction) between two waves scattered along the same path in the opposite directions, and, therefore, shows up already in double scattering. ${ }^{15}$ Since the SPP field is strongly confined in the direction perpendicular to the surface plane, localization phenomena in the elastic SPP scattering can be observed only with near-field techniques. Many features of SPP localization have been clarified, ${ }^{13,16}$ but a proper way of the interpretation of experimental results, i.e., near-field optical images, is yet to be developed and justified. Numerical simulations for various configurations of scatterers and different orders of scattering would certainty be of great help in this case.

SPP scattering by surface inhomogeneities has been considered in many theoretical papers (a good overview can be found in a paper by Pincemin et al. ${ }^{17}$ ). In general, this problem is very complicated, and even a fairly simple case of the SPP scattering, i.e., the scattering of a homogeneous SPP by a circularly symmetric defect on a metal surface, requires elaborate numerical simulations. ${ }^{18}$ The approximation of pointlike dipolar scatterers has been recently used to describe local excitation and scattering of SPP's out of the surface plane $^{19}$ as well as to simulate localization effects. ${ }^{20}$ In the latter work, the indirect coupling between dipoles via the excitation of SPP's has been neglected, thus making the results obtained of limited use. As far as the elastic (in the surface plane) SPP scattering is concerned, we should bear in mind that the scattering observed experimentally ${ }^{12}$ was close to isotropic. Isotropic scatterers have been also pointed out as a limiting case of small scatterers in a rigorous consideration of SPP scattering, ${ }^{18}$ and used in rather simplified simulations of SPP excitation with individual surface defects. ${ }^{5}$ Taking this into account one can approximate a scattered SPP by a cylindrical SPP, which is described by the Hankel function with the lowest angular number $(m=0)$ and with the wave number determined by the same dispersion relation as for a plane SPP. ${ }^{21}$ Such an approach allows one to circumvent the very complicated problem of SPP scattering by surface inhomogeneities and concentrate the efforts on various phenomena that were experimentally observed in (multiple) elastic SPP scattering., ${ }^{4,11-13,16}$

In this paper, we present numerical simulations of elastic SPP scattering by randomly located and specifically configured isotropic pointlike scatterers, and compare our calculations with the previously reported and additionally obtained experimental results. The paper is organized as follows. In Sec. II the main relationships used in our calculations are given and explained. Numerical simulations of single, double, and multiple scattering are presented on Sec. III for randomly situated scatterers showing the interplay between different orders of scattering and localization phenomena. In Sec. IV various optical microcomponents, such as plane and curved micromirrors, formed by sets of point scatterers are considered. The appropriate experimental results are presented and compared with our calculations in Sec. V. Finally, in Sec. VI we summarize the results obtained and offer our conclusions.

\section{MODEL FOR ELASTIC SCATTERING OF SURFACE POLARITONS}

Our model is based on two assumptions, viz., (i) the elastic scattering of SPP's is dominant with respect to the SPP scattering out of the surface plane, and (ii) the SPP scattered by an individual surface defect represents an isotropic cylindrical SPP. The first assumption is justified by the experimental observations showing the appropriate interference patterns, whose periods corresponded to the interference between the incident and scattered SPP's, ${ }^{4,11-13,16}$ and signal (tip-surface) distance dependencies, that exhibited exponential behavior related to the evanescent decay of the SPP field. ${ }^{8}$ In addition, recent theoretical results for an individual surface defect indicated that the elastic SPP scattering becomes dominant over the scattering out of the surface plane in the case of resonant SPP scattering. ${ }^{18}$ The second assumption is primarily based on the circumstance that wellpronounced parabolic interference fringes were observed with SPP scattering by individual defects. ${ }^{4} 12$ Such a pattern corresponds to the interference between the excited SPP with a plane phase front and the scattered SPP with a cylindrical phase front. ${ }^{12}$ Furthermore, it has been theoretically shown that the scattering by a circularly symmetric defect becomes isotropic for small scatterers. ${ }^{18}$

Having substantiated the main two assumptions, we can proceed with the formulation of the scattering problem. Since, in the regime of linear scattering, the field amplitude of a scattered SPP is proportional to the field of an incident SPP at the site of the scatterer, surface defects can be viewed as pointlike scatterers characterized by their effective polarizabilities that relate phenomenologically the scattered field amplitudes to the incident ones. In addition, the geometry of elastic SPP scattering is essentially two-dimensional, i.e., at any surface coordinate $\mathbf{r}$, the total SPP field being a superposition of cylindrical SPP's with the same wave number $\beta$ (equal to that of a plane SPP) ${ }^{21}$ exhibit the same spatial dependence along the direction perpendicular to the surface plane. Therefore, the well-known exponential decays of the SPP field into the neighbor media ${ }^{1}$ will be omitted, and the total SPP field will be represented hereafter by its magnitude $E(\mathbf{r})$ at the surface plane.

Taking into account the aforementioned considerations, the main relation for the total SPP field at an arbitrary surface point $\mathbf{r}$, which does not coincide with the position of scatterers, can be written down as follows:

$$
E(\mathbf{r})=E_{0}(\mathbf{r})+\sum_{j=1}^{N} \alpha_{j} E\left(\mathbf{r}_{j}\right) G\left(\mathbf{r}, \mathbf{r}_{j}\right)
$$


(a)

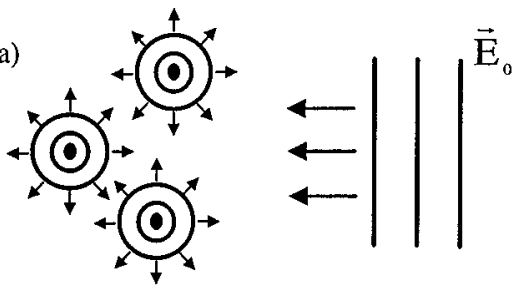

(b)

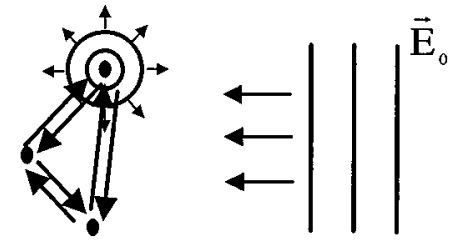

(c)

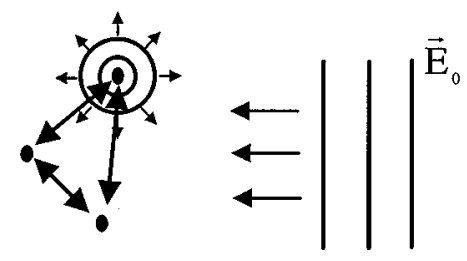

FIG. 1. Schematic representation of different orders of scattering: single (a), double (b), and multiple (c) scattering.

with

$$
G\left(\mathbf{r}, \mathbf{r}_{j}\right)=\frac{i}{4} H_{0}^{(1)}\left(\beta\left|\mathbf{r}-\mathbf{r}_{j}\right|\right)
$$

where $E_{0}$ is the incident SPP field, $\alpha_{j}$ is the effective polarizability of the $j$ th scatterer located at the surface coordinate $r_{j}, N$ is the number of scatterers, $H$ is the Hankel function, and $\beta$ is the SPP propagation constant, which is determined by the dielectric constants of media that are adjacent to the interface. For example, for semi-infinite media on both sides of the air-metal interface $\beta=(2 \pi / \lambda)[\varepsilon /(\varepsilon+1)]^{0.5}$, where $\lambda$ is the wavelength of light in air, and $\varepsilon$ is the dielectric constant of metal. ${ }^{1}$ The effective polarizability $\alpha$ is dimensionless, and corresponds to the scatterer's strength as far as the elastic SPP scattering is concerned. Using the far-field approximation for the Hankel function ${ }^{22}$ it is relatively straightforward to express the total (elastic) cross section $\sigma$ of the scatterer ${ }^{18}$ via the effective polarizability: $\sigma$ $=\alpha^{2} /[4 \operatorname{Re}(\beta)]$. Calculation of the polarizability or the cross section from the actual surface profile of the scatterer is rather complicated, ${ }^{18}$ and, in the following numerical simulations, we shall use the estimate of $\alpha$ based on the experimental near-field optical images. ${ }^{4,12}$

The general relation for the total SPP field [Eq. (1)] is a self-consistent equation of multiple scattering, and can be used only after the self-consistent field at the sites of the scatters $E\left(\mathbf{r}_{j}\right)$ has been determined. The significance of multiple SPP scattering should be considered in relation with the ratio between the SPP propagation length $L=[2 \operatorname{Im}(\beta)]^{-1}$ and the elastic scattering mean free path $l \sim R^{2} / \sigma$, where $R$ is the average separation between scatterers. ${ }^{13}$ If $l>L$, the regime of multiple scattering reduces to the regime of single scattering [Fig. 1(a)], and, consequently, the zeroth-order Born approximation can be used to evaluate the selfconsistent field at the sites of the scatterers: $E^{0}\left(\mathbf{r}_{j}\right)$ $=E_{0}\left(\mathbf{r}_{j}\right)$. As the SPP propagation length increases, the re- gime of single scattering changes to the regime of double scattering [Fig. 1(b)], when the following relation is valid: $2 l>L>l$. In such a case, the first Born approximation should be invoked:

$$
E^{1}\left(\mathbf{r}_{j}\right)=E_{0}\left(\mathbf{r}_{j}\right)+\sum_{l=1, l \neq j}^{N} \alpha_{l} E_{0}\left(\mathbf{r}_{l}\right) G\left(\mathbf{r}_{j}, \mathbf{r}_{l}\right) .
$$

Note that, for randomly situated scatterers, the effect of weak SPP localization would become pronounced already in double scattering. ${ }^{13-15}$

Finally, for $L \gg l$, the regime of multiple SPP scattering prevails [Fig. 1(c)], and the successive Born iterations should be used to calculate the self-consistent field at the scatterers' sites:

$$
E^{n}\left(\mathbf{r}_{j}\right)=E_{0}\left(\mathbf{r}_{j}\right)+\sum_{l=1, l \neq j}^{N} \alpha_{l} E^{n-1}\left(\mathbf{r}_{l}\right) G\left(\mathbf{r}_{j}, \mathbf{r}_{l}\right) .
$$

For randomly situated scatterers, multiple scattering may eventually result in strong SPP localization. ${ }^{13}$ It should be also borne in mind that, in the case of sufficiently strong (resonance) interaction, the Born iterations become divergent, and the exact solution of the self-consistent equation has to be employed. ${ }^{23}$ However, in the conducted numerical simulations, the Born series expansion converged rapidly, and a few iterations [typically, $n<10$ in Eq. (3)] were sufficient to obtain stable values of the self-consistent field at the sites of the scatterers.

Closing the description of our model, we would like to comment on the difference in characteristics between the SPP propagating along the air-metal interface of two semiinfinite media and that of a thin metal layer placed on the surface of a glass prism (this is typical for experimental investigations). The real part of the propagation constant $\beta$ changes only slightly (increases with the decrease of film thickness) for usual thicknesses $(>30 \mathrm{~nm}) .{ }^{1}$ However, the SPP propagation length for a thin metal layer can be considerably less than that for semi-infinite media due to the coupling between the excited SPP and the field components propagating in the prism (the radiation damping). ${ }^{1}$ For the sake of simplicity, we shall use the relations for semi-infinite media while keeping in mind the reduction of $L$ under experimental conditions (due to the finite layer thickness).

\section{RANDOM SCATTERERS}

Elastic SPP scattering by surface roughness and the resulting localization phenomena have been considered in most cases by using direct evaluation of (experimentally obtained) near-field optical images generated due to interference between the excited and scattered SPP's. ${ }^{4,13,16}$ Quite recently, we have introduced the usage of spatial Fourier spectra of near-field optical images for deciphering their content, i.e., for detection of the scattered SPP's and evaluation of the scattering order. ${ }^{8}$ In the following, we shall confirm the previous observations and elucidate the relationship between the Fourier spectrum of intensity distribution and the scattering order by using numerical simulations.

We begin with the estimation of the magnitude of the effective polarizability $\alpha$ for a typical individual scatterer to 


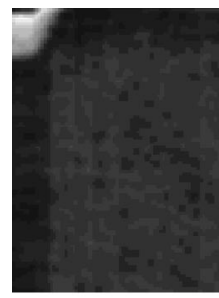

(a)

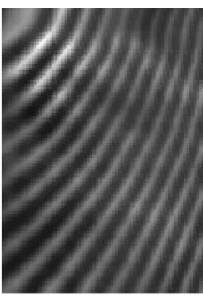

(b)

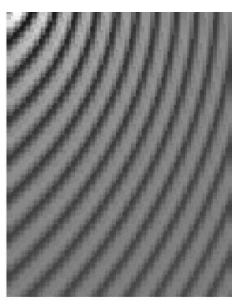

(c)
FIG. 2. Gray-scale topographical (a) and near-field optical (b) experimental images $3 \times 4 \mu \mathrm{m}^{2}$ along with the corresponding representation (c) of the calculated intensity distribution for the effective polarizability $\alpha=3$. The experimental images were obtained with the polariton excited at the wavelength of $633 \mathrm{~nm}$ along the silver film. The depth of the topographical image is $96 \mathrm{~nm}$. Contrast, i.e., the relative difference between maximum and minimum signal, of the near-field optical image recorded with the fiber tip having the contrast correction factor of $\sim 1.2$ is $73 \%$. Contrast in the calculated intensity distribution is $90 \%$.

be used in our calculations. This can be done by considering the characteristic interference pattern obtained experimentally with an individual scatterer and by fitting $\alpha$ so that the calculated intensity distribution would have a similar contrast. One should also remember to take into consideration the contrast correction factor that accounts for spatial frequency filtration performed by a fiber tip. ${ }^{8}$ The result of the described procedure is shown in Fig. 2 for the experiment, in which the SPP excited at the wavelength of $\lambda=633 \mathrm{~nm}$ along the air-metal interface of a silver film (thickness is $\sim 45 \mathrm{~nm}),{ }^{8,12,13}$ and for the simulations with the effective polarizability $\alpha=3$. The propagation constant $\beta$ used in these (and following) simulations has been calculated for semi-infinite media on both sides of the air-metal interface with the metal dielectric constant $\varepsilon=-16+i$, which is a typical value for silver films and the wavelength of $\lambda=633$ nm. ${ }^{8,13}$ The total elastic cross section of the scatterer can be then evaluated as described in the previous section: $\sigma$ $\approx 0.22 \mu \mathrm{m}$. Note that, for a symmetric surface defect considered theoretically, ${ }^{18}$ the same total cross section would correspond (in the first Born approximation) to the scatterer with the height of $0.1 \mu \mathrm{m}$ and the radius of $0.7 \mu \mathrm{m}$. The obtained parameters are similar to those of experimentally observed scatterers. ${ }^{4,12}$

In the following calculations we consider 25 equivalent scatterers with $\alpha=3$, that are placed randomly in the area $5 \times 5 \mu \mathrm{m}^{2}$ and illuminated by a plane wave $(\lambda=633 \mathrm{~nm})$ propagating from the right side toward the left in the horizontal direction. In this case, the propagation length ( $L$ $\sim 23 \mu \mathrm{m}$ ) is sufficiently large in comparison with the elastic scattering mean free path $l \sim R^{2} / \sigma \approx 4.5 \mu \mathrm{m}$. Therefore, by using the Born approximations with different orders as described in the previous section, we can subsequently simulate the regimes of single (Fig. 3), double (Fig. 4), and multiple (Fig. 5) scattering. Experimentally, this would correspond to the usage of different wavelengths or/and different metal films to change the relation between the SPP propagation length and the elastic scattering mean free path. ${ }^{13}$ Note that, in the following, the field intensity distribution in the immediate vicinity of a scatterer will be averaged over the area of $150 \times 150 \mathrm{~nm}^{2}$ centered around the scatterer's location (to

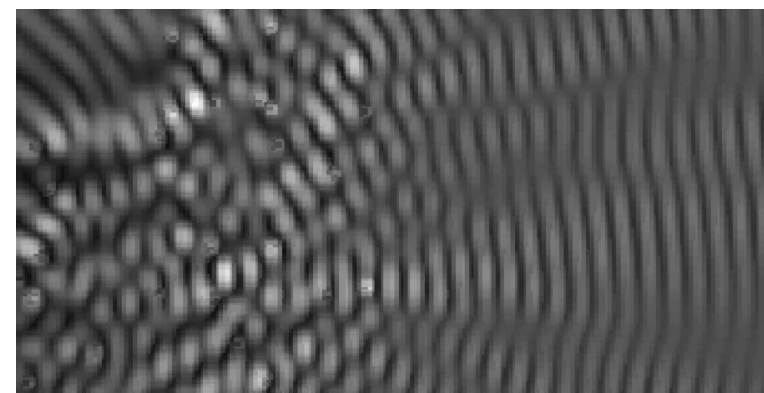

(a)

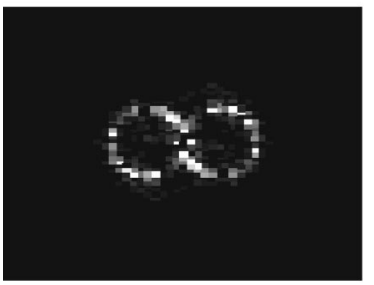

(b)

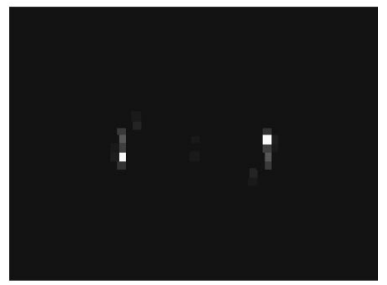

(c)
FIG. 3. Gray-scale representation of the total field-intensity distribution (a) and Fourier spectrum magnitudes corresponding to the intensity distributions inside (b) and outside (c) of the scatterers' area. The intensity distribution within the area of $10 \times 5 \mu \mathrm{m}^{2}$ was calculated in the regime of single scattering by 25 scatters with $\alpha$ $=3$ randomly distributed in the left half. Contrast of the images of $100 \%$.

avoid anomalously large and physically meaningless values of the field intensity). Finally, one should bear in mind that the Fourier spectrum $F(\mathbf{k})$ of an intensity distribution, which is a real function of spatial variables, has Hermitian symmetry and, therefore, its magnitude distribution is symmetric with respect to the origin, i.e., $|F(-\mathbf{k})|=|F(\mathbf{k})|$. For ex-

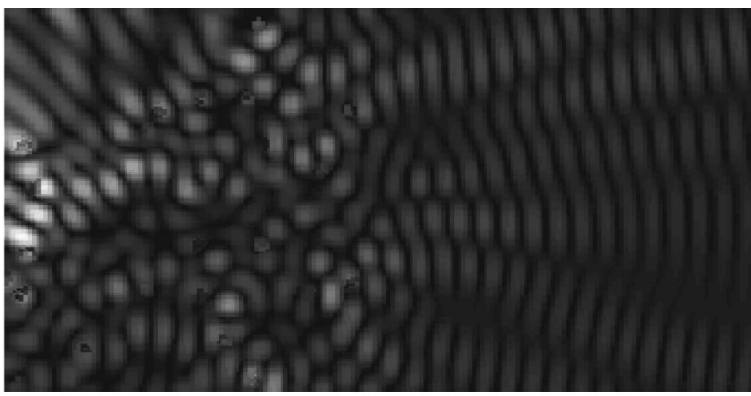

(a)

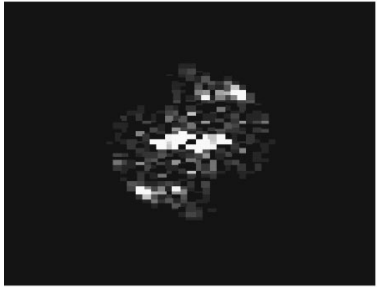

(b)

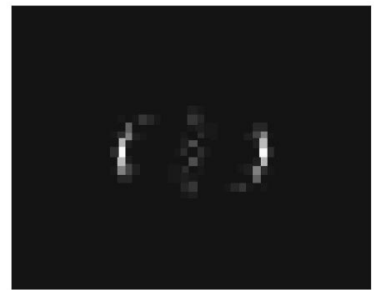

(c)
FIG. 4. Gray-scale representations of the total field-intensity distribution (a) calculated in the regime of double scattering and Fourier spectrum magnitudes corresponding to the intensity distributions inside (b) and outside (c) of the scatterers' area. All else is as in Fig. 3. 


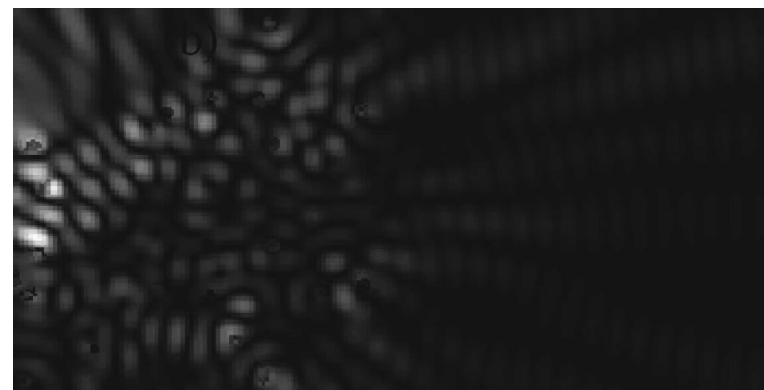

(a)

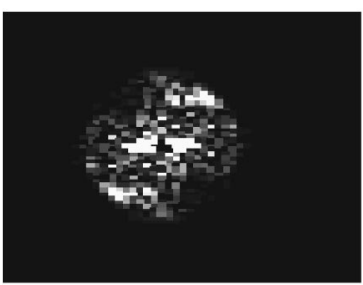

(b)

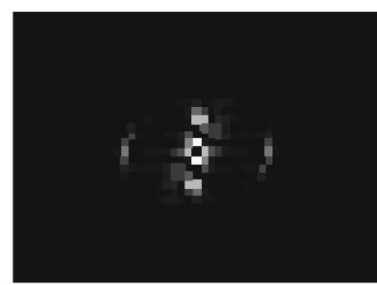

(c)
FIG. 5. Gray-scale representations of the total field-intensity distribution (a) calculated in the regime of multiple scattering and Fourier spectrum magnitudes corresponding to the intensity distributions inside (b) and outside (c) of the scatterers' area. All else is as in Fig. 3.

ample, the intensity interference pattern for two (unequal in amplitude) plane waves with wave vectors $\mathbf{k}_{1}$ and $\mathbf{k}_{2}$ would result in the Fourier spectrum, whose magnitude (besides being nonzero at $\mathbf{k}=0)$ has the same value for $\mathbf{k}= \pm\left(\mathbf{k}_{2}\right.$ $-\mathbf{k}_{1}$ ). In the following, the spectrum magnitude at the origin (that corresponds to the average value of intensity) will be not shown on gray-scale representations of Fourier spectra (to better visualize weak scattered waves).

In the regime of single scattering, i.e., when the zerothorder Born approximation is applied, the intensity distribution reflects the interference between the incident plane wave and the scattered cylindrical waves [Fig. 3(a)]. Consequently, the spatial Fourier spectrum of the intensity distribution in the area, within which the scatterers are contained, represents a pair of open circles with the radius corresponding to the propagation constant $\beta$ [Fig. 3(b)]. ${ }^{8}$ Outside of the scatterers' area, a nearly plane (reflected) wave is formed, and it propagates in the specular direction with respect to some average boundary of the scattering region. The corresponding Fourier spectrum shows two bright spots aligned along the perpendicular to this boundary [Fig. 3(c)].

In the regime of double scattering [the first Born approximation, Eq. (2)], the multiple interference effects become already pronounced: (i) scattered waves inside the scatterers' area are significantly stronger than those outside it [Fig. 4(a)], (ii) their amplitudes are comparable with that of the incident wave thereby resulting in a diffuse Fourier spectrum [Fig. 4(b)], and (iii) outside of the scatterers' area, a backscattered wave (propagating in the direction opposite to that of the incident wave) is formed, and this wave is even stronger than the specularly reflected one [Fig. 4(c)]. These effects become more pronounced in the regime of multiple scattering (Fig. 5) when the successive iterations are used to calculate the self-consistent field at the scatterers' sites [Eq. (3)]. Note that, even though the intensity distribution inside

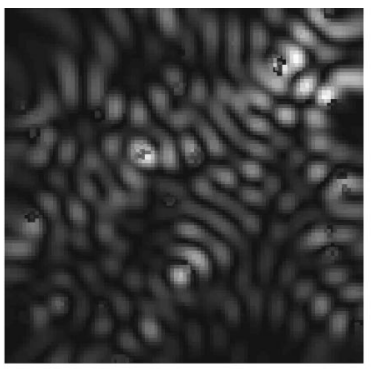

(a)

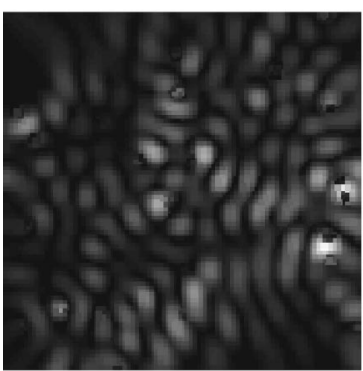

(c)

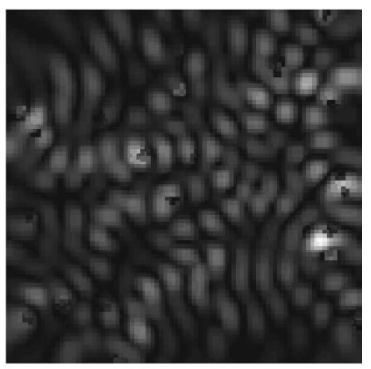

(b)

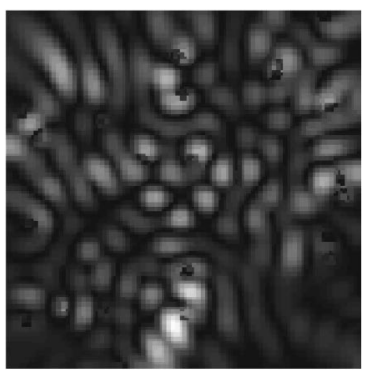

(d)
FIG. 6. Gray-scale representations of the total field intensity distributions in the area of $5 \times 5 \mu \mathrm{m}^{2}$ calculated in the regime of multiple scattering (by 25 scatterers with $\alpha=3$ randomly distributed in the area) for different wavelengths of light: $\lambda=570$ (a), 600 (b), 630 (c), and 660 (d) nm. Contrast of the images is $100 \%$.

the scatterers' area is rather similar to the one for the doublescattering regime [cf. Figs. 5(a) and 4(a)], the corresponding Fourier spectrum contains notably more spatial frequencies [cf. Figs. 5(b) and 4(b)]. Actually, it represents a nearly filled circle with the radius that is twice the propagation constant $\beta$, a feature that is in a complete agreement with the experimental results obtained with SPP scattering on a rough surface of gold film. ${ }^{8}$

Finally, we considered the influence of the wavelength $\lambda$ on the intensity distribution established inside the scatterers' area in the regime of multiple scattering. For the propagation length $L$ being smaller than the lateral extension of the scatterers' area, the corresponding interference pattern should become different when the wavelength is changed by $\delta \lambda$ $\sim \lambda^{2} / L \sim 15 \mathrm{~nm}$. In our case, the area dimension is noticeably smaller than the propagation length, and variations in the intensity distribution (bright spots in particular) were found pronounced for the wavelength variation of $30 \mathrm{~nm}$ (Fig. 6). Such a wavelength dependence is yet another characteristic feature related to interference in multiple scattering. The corresponding spatial Fourier spectra are similar to each other and the one shown in Fig. 5(b). Note that the overall appearance of bright spots (Fig. 6) is quite similar to the bright spots that were observed in experimental studies of SPP scattering by rough metal films and attributed to strong SPP localization. $4,8,13,16$

The presented simulations demonstrate the relationship between different regimes of scattering, spatial Fourier spectra of the field intensity distributions, and localization phenomena. The results obtained are consistent with the previously reported experimental observations. ${ }^{4,8,13,16}$ However, even though the aforementioned bright spots (Fig. 6) are undoubtedly the result of interference in multiple scattering, 
they should not be directly related to strong localization as the size $(d=5 \mu \mathrm{m})$ of the scatterers' area used in our calculations is too small in comparison with the propagation length $(L \sim 23 \mu \mathrm{m})$. Actually, the opposite relation should be satisfied: $d \gg L(\gg l)$. This is hardly a problem in the experiment but, as far as the calculations are concerned, complying with this demand means an increase in number of scatterers to be considered by at least two orders of magnitude. Such extensive calculations are rather time consuming and go beyond the frame of this work.

\section{TWO-DIMENSIONAL MICRO-OPTICS}

The main idea of the technique that we suggested and experimentally demonstrated ${ }^{12}$ is to line up several microscatterers with small separations in order to produce a micromirror. A curved line of scatterers would evidently produce a focusing micromirror, and by using a proper combination of straight and curved mirrors one should then be able to create virtually any conceivable micro-optical circuit. Note that the operation of reflective optical elements is inherently much less sensitive to the light wavelength than that of refractive ones, because in the limit of geometrical optics the former are dispersionless. However, it is also clear from the outset that optical microcomponents, which consist of a few microscatterers and occupy an area of a few wavelengths in size, can exhibit relatively strong wavelength dispersion and configurational instability (i.e., instability with respect to variations in geometrical parameters) in the overall scattering behavior

The original idea was to model the aforementioned microelements by using the same relations [Eqs. (1) and (3)] as in the previous section. However, we found that the calculations performed within the zeroth-order Born approximation fitted somewhat better to the reported experimental results ${ }^{12}$ than more accurate calculations with multiple scattering being taken into account [Eq. (3)]. We believe that this can be explained by the fact that artificially fabricated microscatterers are relatively large (micrometer-sized) ${ }^{12}$ and, therefore, (i) may respond differently (less efficiently) to the strongly divergent scattered wave than to the incident plane wave and/or (ii) may enhance the coupling from the exciting totally internally reflected wave to the SPP (as is the case with individual surface defects created by a direct-write PSTMbased technique $\mathrm{e}^{5,10}$ ). These reasons can be taken into account by introducing an additional term into Eq. (1) that is responsible only for the scattering of the incident (plane) wave:

$$
E(\mathbf{r})=E_{0}(\mathbf{r})+\sum_{j=0}^{N} \alpha_{j} E\left(\mathbf{r}_{j}\right) G\left(\mathbf{r}, \mathbf{r}_{j}\right)+\sum_{j=1}^{N} \alpha_{j}^{*} E_{0}\left(\mathbf{r}_{j}\right) G\left(\mathbf{r}, \mathbf{r}_{j}\right),
$$

where $\alpha_{j}^{*}$ is the auxiliary (effective) polarizability of the $j$ th scatterer accounting for the additional scattering channel, i.e., scattering of the incident field $E_{0}$ that occurs along with multiple scattering [described by Eq. (3)]. Note that the scattering by an individual scatterer shown in Fig. 2 is described by the effective polarizability $\alpha+\alpha^{*}$, and, therefore, we have used the condition $\alpha+\alpha^{*}=3$ (see Sec. III) when comparing the calculated and experimental results.

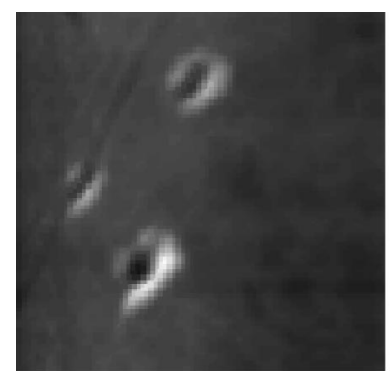

(a)

(c)

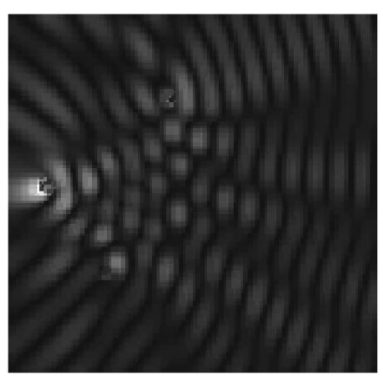

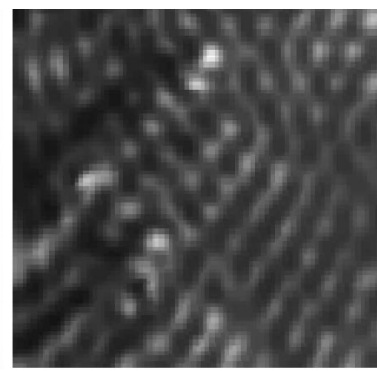

(b)

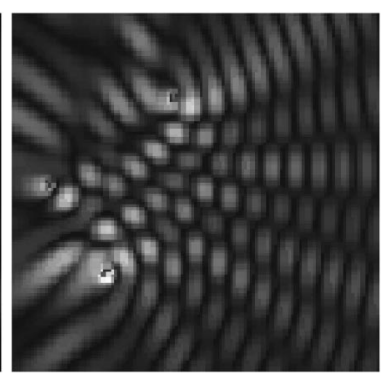

(d)
FIG. 7. Gray-scale topographical (a) and near-field optical (b) experimental images $4 \times 4 \mu \mathrm{m}^{2}$ along with the corresponding representations of the calculated intensity distributions calculated with $\alpha=3, \alpha^{*}=0$ (c), and $\alpha=2, \alpha^{*}=1$ (d). The experimental images were obtained with the polariton excited at the wavelength of 633 $\mathrm{nm}$ along the silver film. The depth of the topographical image is 82 $\mathrm{nm}$. Contrast of the optical images is $90 \%$ (b) and $100 \%$ (c) and (d).

In the following calculations we consider optical microcomponents consisting of equivalent scatterers, that are placed in the area $5 \times 5 \mu \mathrm{m}^{2}$ and illuminated by a plane wave propagating from the right side toward the left in the horizontal direction. Using the experimental results concerning a corner square mirror (the most complicated structure created so far) ${ }^{12}$ we have found a very good agreement between the experimental near-field optical image and the intensity distribution calculated with $\alpha=2$ and $\alpha^{*}=1$ [Eqs. (3) and (4)], whereas the simulations with $\alpha=3$ and $\alpha^{*}$ $=0[$ Eqs. (3) and (1)] resembled poorly the experimental image (Fig. 7). For this reason, the polarizability values obtained $\left(\alpha=2, \alpha^{*}=1\right)$ were used in our simulations of twodimensional optical microcomponents, that are presented hereafter. Finally, in order to elucidate the dispersion effects, two wavelengths of light, viz., 594 and $633 \mathrm{~nm}$, are considered (laser beams at these wavelengths were used for SPP excitation in our experiments presented in the experimental part of the paper).

Simulation results for line micromirrors composed of different numbers of microscatterers are shown in Fig. 8. When viewing the calculated intensity distributions one should remember that the interference fringes due to the excited and specularly reflected wave are parallel to the mirror line (Wiener fringes). We found that, even though it is possible to reflect the incident wave in the specular direction with only 2 (pointlike) scatterers, the efficiency of a 2-scatterer mirror is small, and that there is practically no angular confinement of the reflected wave. Both efficiency and confinement are significantly better for mirrors composed of 6 (and larger number of) scatterers. We found also that, for smaller number of 


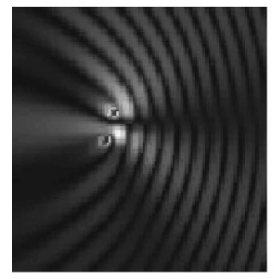

(a)

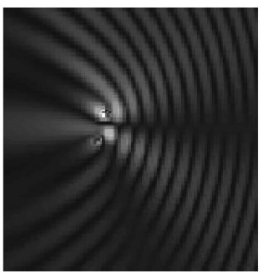

(d)

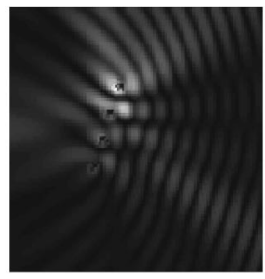

(b)

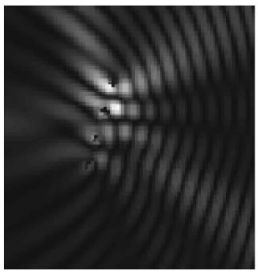

(e)

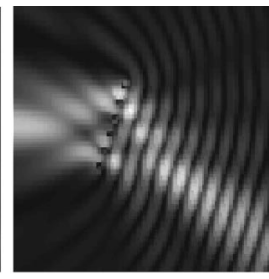

(c)

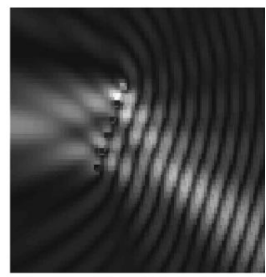

(f)

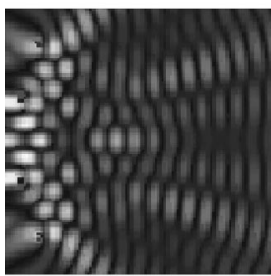

(a)

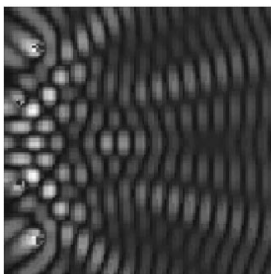

(d)

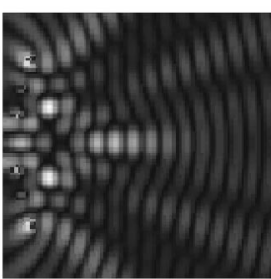

(b)

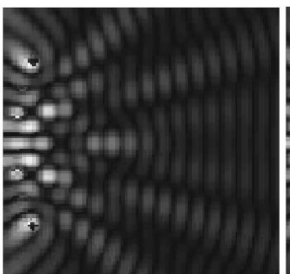

(e)

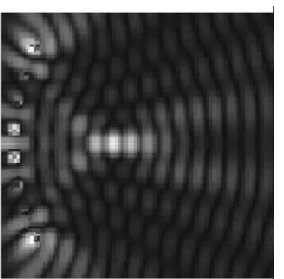

(c)

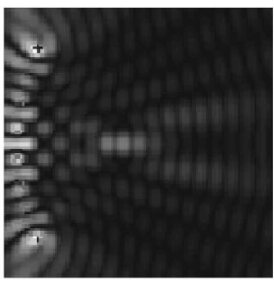

(f)
FIG. 8. Gray-scale representations of the total field-intensity distributions in the area of $5 \times 5 \mu \mathrm{m}^{2}$ calculated (in the regime of multiple scattering) for line micromirrors composed of 2 (a), (d), 4 (b), (e) and 6 (c), (f) scatterers with $\alpha=2, \alpha^{*}=1$, and for two wavelengths of light: $\lambda=633$ (a), (b), (c) and 594 (d), (e), (f) nm. Contrast of the images is $100 \%$.

scatterers, the operation of micromirror is strongly dependent upon geometrical parameters, i.e., mirror orientation and interscatterer distances, as well as the light wavelength. Once the scatterers' number is sufficiently large, the operation becomes stable and the characteristics (efficiency and confinement) approach the expected ones [cf. Figs. 8(c) and 8(f)].

The results of numerical simulations for corner square micromirrors are shown in Fig. 9. The conclusions similar to those formulated above can be deduced for this configuration as well. Apparent on comparison of the interference fringes in intensity distributions for different mirrors is the improvement in the phase distribution of the (back) reflected wave

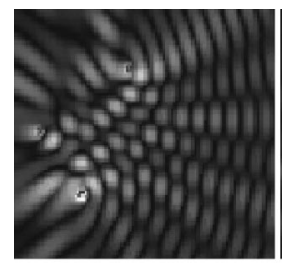

(a)

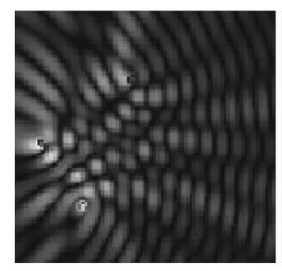

(d)

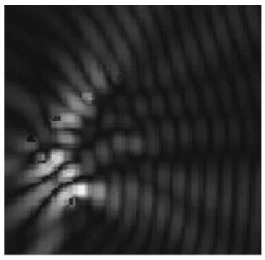

(b)

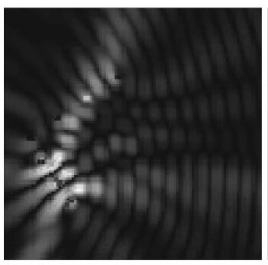

(e)

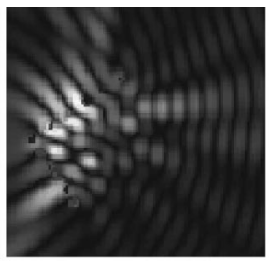

(c)

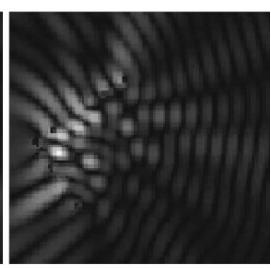

(f)
FIG. 9. Gray-scale representations of the total field-intensity distributions in the area of $5 \times 5 \mu \mathrm{m}^{2}$ calculated for corner square micromirrors composed of 3 (a), (d), 7 (b), (e), and 11 (c), (f) scatterers. All else is as in Fig. 8.
FIG. 10. Gray-scale representations of the total field intensity distributions in the are of $5 \times 5 \mu \mathrm{m}^{2}$ calculated for curved micromirrors (with the focal length of $2 \mu \mathrm{m}$ ) composed of 4 (a), (d), 6 (b), (e), and 8 (c), (f) scatterers. All else is as in Fig. 8.

with the increase in the number of scatterers that compose a micromirror. Actually, the angular confinement of the reflected wave for an 11-scatterer corner mirror is also quite good, but this feature is somewhat obscured in the total fieldintensity distributions because of very bright spots related to interference effects in multiple scattering going on inside the scatterers' area (see Sec. III). Overall behavior of corner micromirrors appears much more complicated than that of line micromirrors (cf. Figs. 8 and 9), thereby warning against designing sophisticated microcomponents.

An ideal focusing micromirror should consist of scatterers placed along a parabolic curve: $y^{2}=4 F x$, where $(x, y)$ is the orthogonal system of coordinates in the surface plane, the $x$ axis is oriented along the optical axis of the mirror, and $F$ is the focal length. Simulations for micromirrors designed to have $F=2 \mu \mathrm{m}$ are shown in Fig. 10. In accordance with the previous observations, the operation becomes a stable and focusing effect-more pronounced with the increase of the scatterers' number. Note that, in the focusing area, the dispersion in the intensity distributions for 8-scatterer mirrors is actually small, but a relatively large difference in the intensity of bright spots (seen inside the scatterers' area) produces an effect of large dispersion in the focusing properties [cf. Figs. 10(c) and 10(f)].

The presented simulations demonstrate the feasibility of creating two-dimensional optical microcomponents by using a specially configured set of scatterers. Both wavelength dispersion and configurational instability of their characteristics are found to decrease rapidly with the increase of the number of scatterers. However, even for a component composed of large number of scatterers, the operation can exhibit unexpected features, e.g., strong field enhancement (bright spots) inside the scatterers' area, which may influence unfavorably the component's characteristics. We believe that the numerical simulations using the appropriate polarizability values (fitted to particular experimental conditions) would help to 


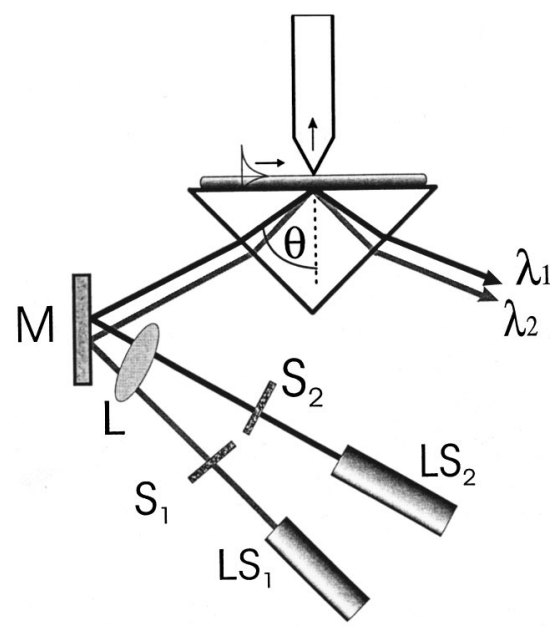

FIG. 11. Schematic of the experimental setup for near-field imaging of intensity distributions of surface plasmon polaritons resonantly excited in turn at two wavelengths $\left(\lambda_{1}=594 \mathrm{~nm}, \lambda_{2}=633\right.$ nm). $L S_{1,2}$, laser sources; $S_{1,2}$ shutters; $L$, lens; $M$, mirror. The polarization of light is parallel to the figure plane.

fabricate the microcomponents with desirable characteristics. Our model seems reasonably simple and accurate to serve adequately this purpose.

\section{EXPERIMENTAL RESULTS}

The experimental setup used in this work consists of a stand alone PSTM combined with a shear force based feedback system and an arrangement for SPP excitation in the usual Kretschmann configuration, and is described in detail elsewhere. ${ }^{4}$ The only modification introduced is the incorporation of an additional laser source in the arrangement used for the SPP excitation (Fig. 11). Thereby, SPP's can be resonantly excited at either of two optical wavelengths, viz., 594 (yellow) and 633 (red) nm. Two samples were used in our experiments presented here: with the silver and gold thin films. A 45-nm-thick silver film has been thermally evaporated on a glass prism (refractive index $n \approx 1.52$ ) in vacuum $\left(\sim 10^{-5}\right.$ Torr $)$ during a rather short time interval $(\sim 10 \mathrm{~s})$ aiming at fabrication of a smooth but relatively soft film [suitable for the experiments on micro-optics of SPP's (Ref. 12)]. A 40-nm-thick gold film has been evaporated (vacuum $\sim 10^{-6}$, time $\left.\sim 30 \mathrm{~s}\right)$ on the base of a prism $(n \approx 1.52)$ that has been covered with a sublayer of colloid gold particles (diameter $\sim 40 \mathrm{~nm}$ ) dried up in atmosphere (suitable for the experiments on multiple SPP scattering ${ }^{8}$ ).

The $p$-polarized (electric field is parallel to the plane of incidence) light beam from either of two He-Ne lasers ( $P$ $\approx 3 \mathrm{~mW}$ ) is weakly focused onto the base of either of two prisms with the metal films (local length $\approx 500 \mathrm{~mm}$, spot size $\sim 400 \mu \mathrm{m})$. Such an arrangement allows one to increase a local intensity of the incident beam while keeping it within the angular width of resonant SPP excitation. The reflected light is detected by a photodiode, and the SPP excitation is recognized as a minimum in the angular dependence of the reflected light power (attenuated total reflection minimum). ${ }^{1}$ The appropriate angular dependencies were measured for both films and both wavelengths in order to determine the resonant excitation angles. By fitting the measured dependencies to those calculated for the three-layer structure, one can determine the film dielectric constants and the relevant SPP characteristics. ${ }^{1}$ The SPP local field is probed with an etched (55 min. in a $40 \%$ solution of hydrofluoric acid) fiber tip that ensures both strong optical signal ${ }^{13}$ and relatively faithful imaging. ${ }^{8,24}$ The SPP excitation exhibited a wellpronounced resonance behavior with the detected optical signal (at resonance) of $\sim 1-10 \mathrm{nW}$ for the silver film, and $0.1-1 \mathrm{nW}$ for the gold film. It should be mentioned that the signal related to the SPP excited at $633 \mathrm{~nm}$ was usually stronger than that connected with the wavelength of $594 \mathrm{~nm}$. Finally, it should be noted that all images presented here are oriented in the way that the excited SPP propagates from the right side toward the left in the horizontal direction.

The average optical signal for both films and both wavelengths decreased exponentially (as expected) with the increase of the tip-surface distance. The observed distance dependence confirms that the near-field optical images represent the SPP intensity distributions, ${ }^{8}$ and, at the same time, indicates the possibility of inducing topographical artifacts in images obtained with shear force feedback (in constant tip-surface distance mode). ${ }^{25}$ We have carefully explored such a possibility by comparing optical images recorded with the silver film for both wavelengths in con stant distance and constant height modes (Fig. 12). The only difference found between these images was related to the displacement of the fiber tip when getting out of the contact with the sample surface. This means that the contrast observed in near-field images is pure optical, i.e., not induced by topographical variations, ${ }^{25}$ a fact that can be explained by rather strong and rapid variations of the near-field intensity in the surface plane. Once this fact is established, the nearfield imaging in constant distance mode is preferable, since it allows one to achieve the best spatial resolution (which deteriorates with the increase of the tip-surface distance). This mode helps also to keep track on the imaged area when successive images of the same area are being taken and, thereby, to account for eventual drift of the sample with respect to the fiber tip. Actually, the stability of our setup appeared rather high, a circumstance that enabled us to obtain the near-field optical images in turn for the two wavelengths at the same surface area (Fig. 12).

\section{A. Random scatterers}

Topographical images of the silver film showed a smooth surface with rarely spaced bumps in the center region, and somewhat more rough surface in the outer region. The corresponding near-field optical images of the SPP intensity distributions at both wavelengths exhibited well pronounced interference patterns, whose dispersion was especially noticeable for rough surface areas (Fig. 13). This indicates that, in the center part of the film, scatterers are situated far away from each other (for example, no scatters are seen on the corresponding topographical image in Fig. 13), and the regime of SPP scattering in this region is close to that of single scattering. In the outer region, the SPP propagation length $(\sim 15 \mu \mathrm{m}$ for both wavelengths and with the radiation damping being taken into account) is apparently larger 

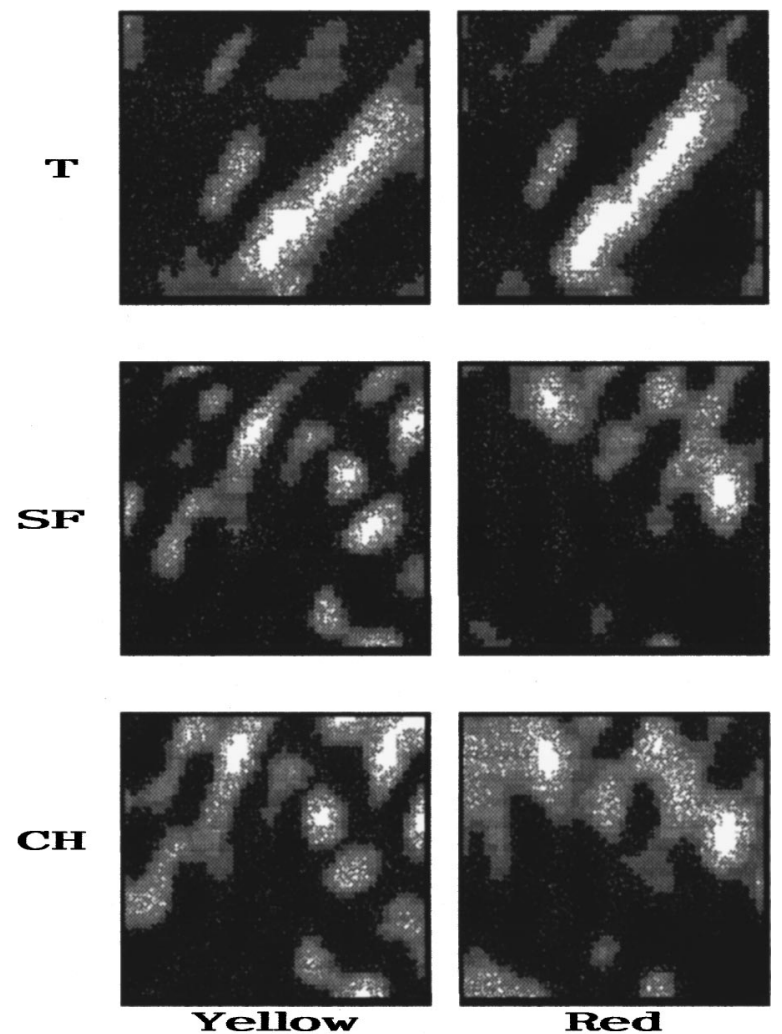

FIG. 12. Gray-scale topographical $(T)$ and near-field optical images $1.8 \times 1.8 \mu \mathrm{m}^{2}$ obtained with the silver film for two wavelengths: $594 \mathrm{~nm}$ (yellow) and $633 \mathrm{~nm}$ (red), when operating the microscope with shear force feedback (SF) and in constant height mode $(\mathrm{CH})$ with the tip-surface distance of $\sim 15 \mathrm{~nm}$. The depth of the topographical images is $\sim 30 \mathrm{~nm}$. Contrast of the optical images is $\sim 95 \%$.

than the elastic scattering mean free path, and multiple scattering results in bright spots whose position is wavelength dependent (see Sec. III).

Topographical images of the gold film showed a rough surface revealing the sublayer of randomly located gold particles (or particle clusters). ${ }^{8}$ The elongated appearance of the
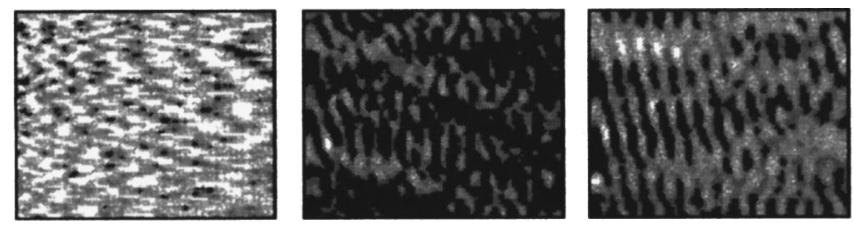

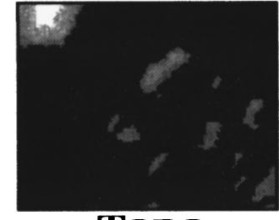

Topo

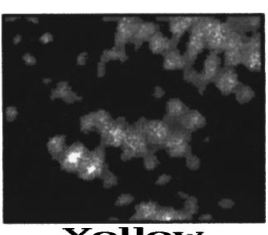

Yellow

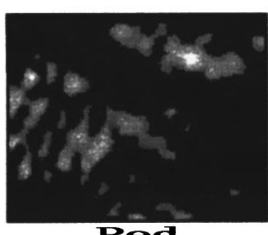

Red
FIG. 13. Gray-scale topographical (Topo) and near-field optical images $4.5 \times 3.6 \mu \mathrm{m}^{2}$ obtained at smooth and rough regions of the silver film for two wavelengths: $594 \mathrm{~nm}$ (yellow) and $633 \mathrm{~nm}$ (red), when operating the microscope with shear force feedback. The depth of the topographical images is $\sim 10 \mathrm{~nm}$ (top set of images) and $92 \mathrm{~nm}$ (lower set). Contrast of the optical images is $\sim 95 \%$.
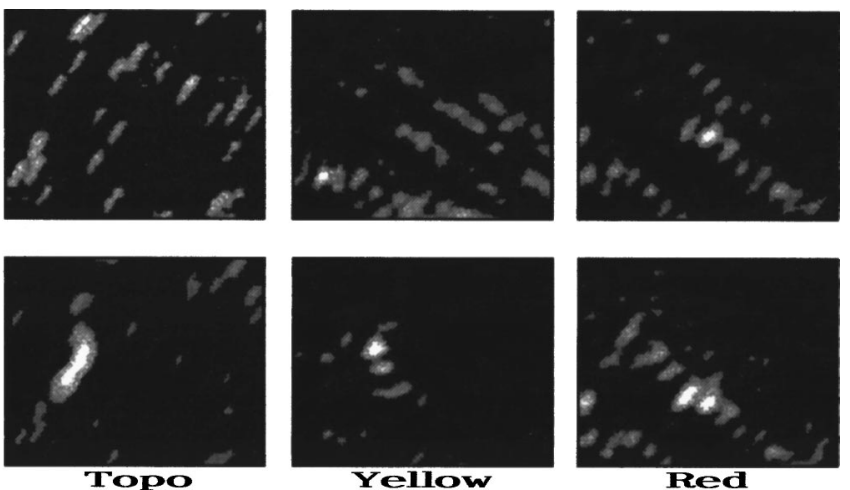

Yellow

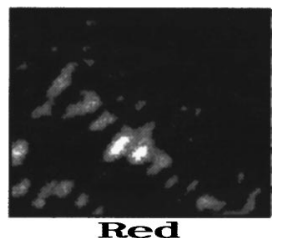

FIG. 14. Gray-scale topographical and near-field optical images obtained with the gold film before (top set of images) and after (lower set) surface modification (the tip was pressed against the surface to the left from the center of the scan area). The depth of the topographical images is $\sim 42 \mathrm{~nm}$ (top set) and $101 \mathrm{~nm}$ (lower set). Contrast of the optical images is $\sim 98 \%$. Everything else is as in Fig. 13.

particles (Fig. 14) is probably induced by an asymmetrical shape of the fiber tip (taking the relatively large cone-angle into account) and/or a tilt of the fiber axis with respect to the normal direction (to the film surface). Such an asymmetry in topographical imaging has also been observed in the experiments with latex spheres placed on a crystal surface, which have been imaged with a similar fiber tip. ${ }^{26}$ The corresponding near-field optical images of the SPP intensity distributions exhibited rather bright spots, that are almost round in shape and located differently for different wavelengths (Fig. 14). These features are typical for the regime of multiple scattering that can eventually result in strong SPP localization. ${ }^{4,8,13}$ An interference pattern produced by multiple scattering is very sensitive to variations in any parameter (wavelength, phase distribution, positions of scatterers, etc.). The sensitivity with respect to scatterers' locations was directly observed in our experiments. The two sets of images shown in Fig. 14 have been taken at the same place of the gold film, whose topography has been modified (after obtaining the top set but before recording the lower set) by pressing the fiber tip against the film surface. It is clearly seen that, after such a modification, the near-field optical images changed drastically (and differently) for both wavelengths.

Spatial frequency spectra of near-field optical images were found (Ref. 8 and Sec. III) particularly useful for establishing the regime of scattering. The spatial spectra of the images presented here (Figs. 13 and 14) are quite similar for the two wavelengths used but different for the surfaces inspected. The spatial Fourier spectrum for the center (smooth) region of the silver film represents a pair of open circles with the radius determined by the SPP propagation constant as should have been expected for the regime of single scattering [cf. Figs. 3(b) and 15(a)]. Spatial spectra for relatively rough areas of the silver and gold films [Figs. 15(b) and 15(c)] contain notably more spatial frequencies approaching the spectrum that corresponds to the developed multiple scattering, i.e., a filled circle with the radius twice the propagation constant [Fig. 5(b)]. 


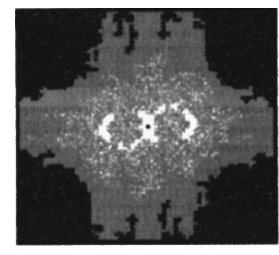

(a)

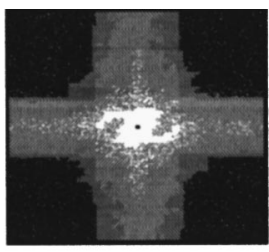

(b)

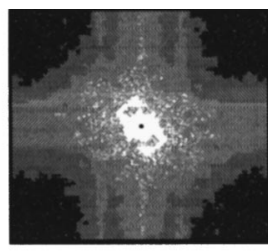

(c)
FIG. 15. Gray-scale logarithmic representations of Fourier spectrum magnitudes for the near-field optical images (Figs. 13 and 14) recorded at smooth (a) and rough (b) regions of the silver film and with the gold film before modification (c).

\section{B. Microcomponents for SPP's}

The technique that has been suggested for fabrication of microcomponents for SPP's ${ }^{12}$ is rather simple: an uncoated fiber tip has to be pressed against a metal film surface. It means that the sample should be moved toward the fiber tip sufficiently fast in comparison with the time response of the shear force feedback system ( $\sim 1 \mathrm{~ms}$, in our case). In the first experiments, the fiber tip has been moved with the piezoelectric translator controlled manually. ${ }^{12}$ In the present experiments, we used a piezodriver (Physik Instrumente, Germany) whose output can be controlled with an external analog signal. We have constructed a simple $R C$-circuit with an adjustable power supply, that delivers voltage pulses (duration $\sim 10 \mathrm{~ms}$ ) to the input of piezo driver. After a few trial experiments we found that the voltage pulses of $\sim 1.5 \mathrm{~V}$ result in the microscatterers of a proper size (similar to that obtained in the preliminary experiments ${ }^{12}$ ). Note that, even though microscatterers with nearly the same efficiency could
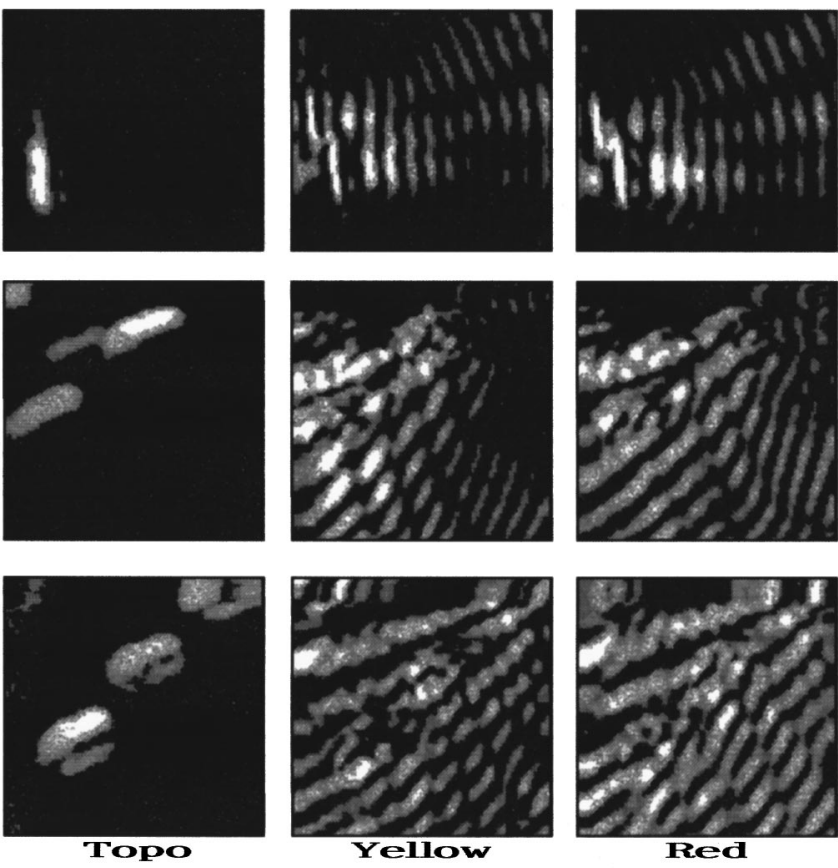

FIG. 16. Gray-scale topographical (Topo) and near-field optical images $4.4 \times 4.3 \mu \mathrm{m}^{2}$ obtained with differently oriented 3-scatterer micromirrors (fabricated on the silver film) for two wavelengths: $594 \mathrm{~nm}$ (yellow) and $633 \mathrm{~nm}$ (red). The depth of the topographical images is $\sim 47 \mathrm{~nm}$ (top set of images), $142 \mathrm{~nm}$ (middle set), and 90 $\mathrm{nm}$ (lower set). Contrast of the optical images is $\sim 100 \%$.
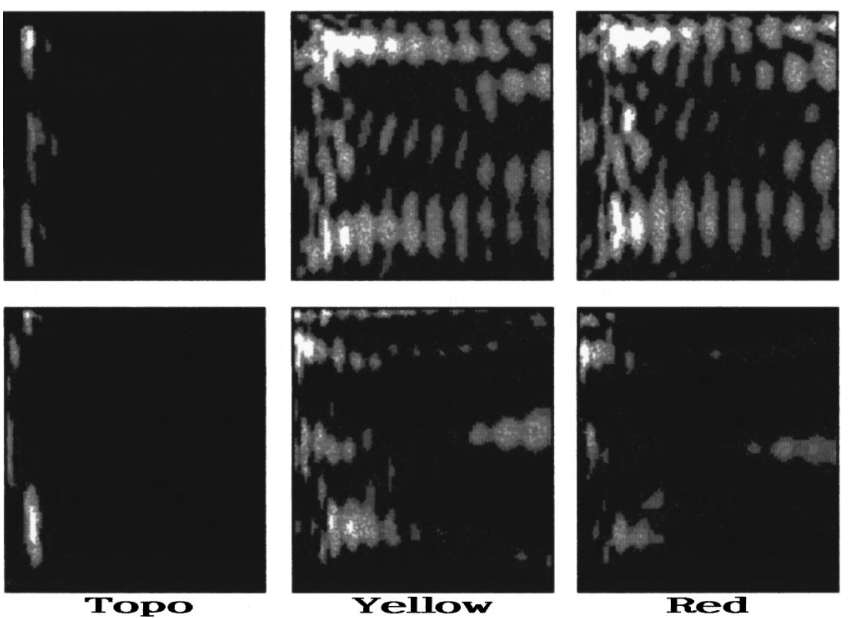

FIG. 17. Gray-scale topographical and near-field optical images obtained with straight (top set of images) and curved (lower set) micromirrors. The depth of the topographical images is $\sim 81 \mathrm{~nm}$ (top set) and $74 \mathrm{~nm}$ (lower set). Contrast of the optical images is $\sim 100 \%$. Everything else is as in Fig. 16.

have been produced in both gold and silver films, the silver film was found more suitable in many respects, e.g., it allows for more efficient SPP excitation and longer SPP propagation length. Therefore, the results presented hereafter are related to the experiments with the silver film.

The wavelength dispersion of microcomponents is the main issue checked out in the experiments presented here. Several 3-scatterer micromirrors oriented differently were fabricated, and their operation for the two wavelengths was investigated (Fig. 16). Similar to our numerical simulations discussed above, the wavelength dispersion of experimentally studied micromirrors appeared noticeable (Wiener fringes look more convincing for SPP's excited by red light) but seemingly insignificant for practical purposes. However, even in this refined experiment, the fabricated micromirrors exhibited the behavior that was not as perfect as one would have liked it to be. Although the reasons may be many, we believe that the nonlinearity of piezotranslators positioning the fiber tip and the asymmetrical shape of the fiber tip should be pointed out. The first reason makes the exact tip positioning difficult, especially when accompanied by the temporal drift of the sample with respect to the tip. The second reason forces one to properly adjust the orientation of the fiber tip (by rotating it around the fiber axis) for the fabrication of a particularly tilted mirror (Fig. 16).

The comparison of operation of the (supposedly) straight and curved line mirrors can serve as a serious check of the developed technique. We have attempted to produce a 3scatterer straight mirror reflecting backward and a 4-scatterer curved mirror focusing the reflected light at $\sim 4 \mu \mathrm{m}$ distance from the mirror (see Sec. IV). The corresponding experimental results are shown in Fig. 17. It is seen that we failed to perfectly align 3 microscatterers (along a straight line), and the reflected waves were quite inhomogeneous for both wavelengths. The focusing micromirror came out well, and the experimentally obtained intensity distributions were rather similar to the simulated ones (compare Figs. 10 and 17 , taking into account the difference in the focal distances). 


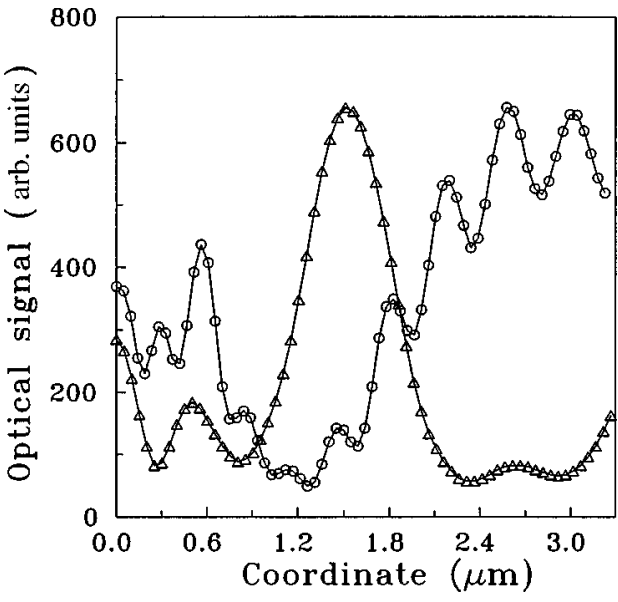

FIG. 18. Horizontal (circles) and vertical (triangles) cross sections of the near-field optical image, that was recorded for the curved micromirror with yellow light (Fig. 17). The horizontal cross section is along the optical axis of the micromirror, and the vertical one is across the focusing area. Solid lines connecting the experimental points serve to guide the eye.

As far as the wavelength dispersion of the straight and curved micromirrors is concerned, the plane-reflected SPP is somewhat more homogeneous for yellow light, but the spatial confinement of the focused reflected SPP looks better for red light (Fig. 17)

Cross sections of the recorded intensity distributions (similar for the two wavelengths) exhibit more than 10 times signal enhancement in the focusing region, whose total width is $\sim 1 \mu \mathrm{m}$ (Fig. 18). Because of the limited size of the image, the length of the focusing area (along the optical axis) can be only approximately estimated as a few micrometers. In addition, the cross section along the optical axis allows one to evaluate the magnitude of the focused SPP field as compared to that of the incident (from the right side toward the left) SPP. Indeed, the contrast of interference fringes in the focusing area (the difference between the maximum and minimum signals divided by their sum) is $\sim \frac{1}{9}$ (Fig. 18). This means that the ratio between the field magnitudes of focused (backscattered) and incident SPP's is $\sim 18$. Taking into account that the incident field is already enhanced at the surface due to the resonant SPP excitation, ${ }^{1}$ one can conclude that the total field enhancement created by focusing the excited SPP can easily reach several orders of magnitude. We believe that the results obtained with the focusing micromirror are rather promising with respect to the possibility of creating strong local-field enhancement at a given place, an effect that can be advantageously exploited, for example, in spatially resolved Raman spectroscopy.

The near-field images look quite similar to the results of numerical simulations with respect to both the overall appearance and wavelength dispersion. In particular, a very good agreement between the experimental and calculated results has been demonstrated for a corner square mirror (Fig. 7). It seems though that the scatterers' number in calculations should be, in general, larger than the experimentally used one for such an agreement to be achieved (cf. Figs. 8 and 16, 10 and 17). There is also the asymmetry in scatterer's shape (and in the scattering properties), which was ob- served previously, ${ }^{12}$ as well as in these experiments, and that is not reflected in our model. One way to circumvent these inconsistencies would be to simulate an experimentally fabricated microscatterer by two pointlike scatterers, which are closely placed along a specific direction (but that do not interact with each other). Another way to tackle this problem is to approximate a scattered SPP by the Hankel function with the angular number $m>0$, i.e., to introduce an anisotropic response of a (pointlike) scatterer. However, the corresponding modifications would bring our model on the next level of complexity and thereby would require significant efforts for these modifications to be properly incorporated in the developed framework. We shall explore these possibilities in our future work.

\section{CONCLUSIONS}

Elastic scattering of SPP's by randomly situated and artificially fabricated microscatterers has been investigated both theoretically and experimentally. Microscatterers have been considered as (two-dimensional) isotropic pointlike scatterers characterized by the effective polarizability, which is directly related to the total (elastic) scattering cross section. For randomly situated scatterers, the results of numerical simulations of different scattering regimes (single, double, and multiple scattering) have been presented illustrating the interplay between different orders of scattering and localization phenomena. The importance of spatial Fourier spectra of SPP intensity distributions for their interpretation has been pointed out. The results of our simulations have been found to be in agreement with the experimental results presented here and in our previous papers. ${ }^{8,13,16}$ Overall, we believe that a certain understanding of elastic SPP scattering by random scatterers and accompanying localization phenomena has emerged and that this allows one to approach other fascinating issues related to SPP scattering, e.g., phenomena associated with photonic band gaps in the optical regime using SPP's. ${ }^{27}$

Various optical microcomponents formed by sets of point scatterers have also been simulated, and the stability and dispersion of their characteristics have been discussed. The appropriate experimental results obtained for straight and curved micromirrors have been presented (for two excitation wavelengths) and compared with the numerical results, demonstrating that the model developed can be successfully used for the design of components for micro-optics of SPP's. A component for micro-optics of SPP's viz., a focusing micromirror, has been experimentally investigated for the first time to our knowledge, and the field enhancement by $\sim 18$ times with respect to the excited SPP has been observed in the focusing area. It is relatively straightforward to combine such a component with other microcomponents, i.e., line and corner square micromirrors, ${ }^{12}$ and eventually with spatially resolved SPP excitation. ${ }^{9,10}$ Overall, the conducted investigations have demonstrated that, by using the developed nearfield techniques and numerical modeling, it is possible to design and fabricate various integrated microcircuits for SPP's (with desirable characteristics) that could have numerous potential applications in spatially resolved (linear and nonlinear) spectroscopy, surface chemical and biosensing, and, in general, in surface optics. 
${ }^{1}$ Surface Polaritons, edited by V. M. Agranovich and D. L. Mills (North-Holland, Amsterdam, 1982); H. Raether, Surface Plasmons, Springer Tracts in Modern Physics, Vol. 111 (Springer, Berlin, 1988).

${ }^{2}$ Near Field Optics, edited by D. W. Pohl and D. Courjon (Kluwer, The Netherlands, 1993).

${ }^{3}$ M. Specht, J. D. Pedarnig, W. M. Heckl, and T. W. Hänsch, Phys. Rev. Lett. 68, 476 (1992); O. Marti, H. Bielefeldt, B. Hecht, S. Herminghaus, P. Leiderer, and J. Mlynek, Opt. Commun. 96, 225 (1993); P. Dawson, F. de Fornel, and J.-P. Goudonnet, Phys. Rev. Lett. 72, 2927 (1994); D. P. Tsai, J. Kovacs, Z. Wang, M. Moskovits, V. M. Shalaev, J. S. Suh, and R. Botet, ibid. 72, 4149 (1994); R. B. G. de Hollander, N. F. van Hulst, and R. P. H. Kooyman, Ultramicroscopy 57, 263 (1995); Y.-K. Kim, P. M. Lundquist, J. A. Helfrich, J. M. Mikrut, G. K. Wong, P. R. Auvil, and J. B. Ketterson, Appl. Phys. Lett. 66, 3407 (1995); Y.-K. Kim, J. B. Ketterson, and D. J. Morgan, Opt. Lett. 21, 165 (1996).

${ }^{4}$ S. I. Bozhevolnyi, I. I. Smolyaninov, and A. V. Zayats, Phys. Rev. B 51, 17916 (1995).

${ }^{5}$ I. I. Smolyaninov, D. L. Mazzoni, J. Mait, and C. C. Davis, Phys. Rev. B 56, 1601 (1997).

${ }^{6}$ R. C. Reddick, R. J. Warmack, and T. L. Ferrel, Phys. Rev. B 39, 767 (1989); D. Courjon, K. Sarayeddine, and M. Spajer, Opt. Commun. 71, 23 (1989); F. de Fornel, J. P. Goudonnet, L. Salomon, and E. Lesniewska, Proc. SPIE 1139, 77 (1989).

${ }^{7}$ D. Van Labeke and D. Barchiesi, J. Opt. Soc. Am. A 10, 2193 (1993); R. Carminati and J.-J. Greffet, Opt. Commun. 116, 316 (1995)

${ }^{8}$ V. Coello, S. I. Bozhevolnyi, and F. A. Pudonin, Proc. SPIE 3098, 536 (1997).

${ }^{9}$ B. Hecht, H. Bielefeldt, L. Novotny, Y. Inouye, and D. W. Pohl, Phys. Rev. Lett. 77, 1889 (1996); B. Hecht, D. W. Pohl, and L. Novotny, in Optics at the Nanometer Scale, edited by M. NietoVesperinas and N. García (Kluwer, Dordrecht, 1996), p. 151.

${ }^{10}$ I. I. Smolyaninov, D. L. Mazzoni, and C. C. Davis, Phys. Rev. Lett. 77, 3877 (1996).

${ }^{11}$ J. R. Krenn, R. Wolf, A. Leitner, and F. R. Aussenegg, Opt. Commun. 137, 46 (1997).

${ }^{12}$ S. I. Bozhevolnyi and F. A. Pudonin, Phys. Rev. Lett. 78, 2823 (1997).
${ }^{13}$ S. I. Bozhevolnyi, Phys. Rev. B 54, 8177 (1996).

${ }^{14}$ B. Souillard, in Chance and Matter, edited by J. Souletie, J. Vannimenus, and R. Stora (North-Holland, Amsterdam, 1987), p. 305; S. John, in Scattering and Localization of Classical Waves in Random Media, edited by P. Sheng (World Scientific, Singapore, 1990), p. 1.

${ }^{15}$ M. P. van Albada, M. B. van der Mark, and A. Lagendijk, in Scattering and Localization of Classical Waves in Random Media (Ref. 14), p. 97; Yu. N. Barabanenkov et al., in Progress in Optics, edited by E. Wolf (Elsevier, New York, 1991), Vol. 29, p. 65 .

${ }^{16}$ S. I. Bozhevolnyi, B. Vohnsen, I. I. Smolyaninov, and A. V. Zayats, Opt. Commun. 117, 417 (1995); S. I. Bozhevolnyi, A. V. Zayats, and B. Vohnsen, in Optics at the Nanometer Scale (Ref. 9), p. 163; S. I. Bozhevolnyi, B. Vohnsen, A. V. Zayats, and I. I. Smolyaninov, Surf. Sci. 356, 268 (1996).

${ }^{17}$ F. Pincemin, A. A. Maradudin, A. D. Boardman, and J.-J. Greffet, Phys. Rev. B 50, 15261 (1994).

${ }^{18}$ A. V. Shchegrov, I. V. Novikov, and A. A. Maradudin, Phys. Rev. Lett. 78, 4269 (1997).

${ }^{19}$ L. Novotny, B. Hecht, and D. W. Pohl, J. Appl. Phys. 81, 1798 (1997).

${ }^{20}$ M. Xiao, A. Zayats, and J. Siqueiros, Phys. Rev. B 55, 1824 (1997).

${ }^{21}$ V. A. Kosobukin, Phys. Solid State 35, 457 (1993); P. J. Valle, E. M. Ortiz, and J. M. Saiz, Opt. Commun. 137, 334 (1997).

${ }^{22}$ Handbook of Mathematical Functions, 9th ed., edited by M. Abramowitz and I. A. Stegun (Dover, New York, 1972).

${ }^{23}$ O. Keller, M. Xiao, and S. Bozhevolnyi, Surf. Sci. 280, 217 (1993).

${ }^{24}$ S. I. Bozhevolnyi, B. Vohnsen, E. A. Bozhevolnaya, and S. Berntsen, J. Opt. Soc. Am. A 13, 2381 (1996).

${ }^{25}$ B. Hecht, H. Bielefeldt, Y. Inouye, and D. W. Pohl, J. Appl. Phys. 81, 2492 (1997); S. I. Bozhevolnyi, J. Opt. Soc. Am. B 14, 2254 (1997).

${ }^{26}$ B. Vohnsen and S. I. Bozhevolnyi, Opt. Commun. 148, 331 (1998).

${ }^{27}$ S. C. Kitson, W. L. Barnes, and J. R. Sambles, Phys. Rev. Lett. 77, 2670 (1996). 